\title{
Hypersensitivity Reaction to Misoprostol—A Case Report
}

\author{
Monika Madaan*, Manju Puri, Ritu Sharma, Shubha Sagar Trivedi \\ Lady Hardinge Medical College (LHMC) \& Srimati Sucheta Kripalini Hospital (SSKH), New Delhi, India. \\ Email: "monikarajivgaur@rediffmail.com
}

Received January 20 $0^{\text {th }}, 2012$; revised February 24 $4^{\text {th }}$ 2012; accepted March 27 $7^{\text {th }}, 2012$

\begin{abstract}
Misoprostol has been used in obstetrics for a number of years in all trimesters of pregnancy. Apart from its side effect of causing congenital malformations in offsprings of users who have unsuccessfully used it as an abortifacient, it is considered a safe drug with few side effects. We here report a case of severe hypersensitivity reaction to misoprostol in a patient where it was used for first trimester abortion in a case of missed abortion.
\end{abstract}

Keywords: Misoprostol; Hypersensitivity; Missed Abortion

\section{Introduction}

Misoprostol has been widely used in obstetrics as a treatment of missed abortion, incomplete abortion, cervical preparation before surgical evacuation, induction of labor and postpartum hemorrhage. It acts through its effect on cervix as a ripening agent and as a uterotonic agent. Misoprostol can be administered by oral, sublingual, vaginal and rectal routes. The rate of absorption by sublingual route is faster as compared to vaginal administration. Sublingual and vaginal routes of administration result in longer lasting levels of misoprostol in plasma than oral administration. Vaginal administration results in regular uterine contractions as compared to oral route. However it is associated with wider variation in absorption rates [1].

In April 2002, FDA finally approved a new label for use of misoprostol during pregnancy [2]. This revises the contraindication and the precaution that misoprostol should not be used in pregnant women by stating that the contraindication is only for pregnant women who are using the medication to reduce the risk of NSAID-induced stomach ulcers. Misoprostol is now a part of the FDA-approved regime for use with mifepristone to induce abortion in early pregnancy and is also recognized for its use for induction of labor.

Misoprostol is considered a safe drug with very few side effects. Common side effects are diarrhea, abdominal pain, headache, menstrual cramps, nausea, chills, shivering and fever. Congenital anomalies have been reported in babies of women who have unsuccessfully used misoprostol as an abortifacient. Few reports of Clostridium sordeilli infection causing septic shock in women undergoing abortion with misoprostol have been described in the literature. We describe a case of hypersensitivity re-

\footnotetext{
*Corresponding author.
}

action with misoprostol.

\section{Case Report}

A 32 year primigravida presented at 12 weeks of gestation with missed abortion. On per vaginal examination cervix was uneffaced, internal os was closed, uterus was anteverted, 10 weeks size, soft and mobile. She was planned for medical abortion and misoprostol $800 \mu \mathrm{g}$ was inserted intravaginally. After 20 minutes of intravaginal placement of misoprostol, patient developed shivering, intense burning sensation and feeling of warmth over face, hands and feet. On examination, pulse rate was 110/ minute, BP was 130/90 mm Hg, flushing was present over ears and there was palmar and plantar erythema. On local examination there was redness over vulval region. Misoprostol was removed from the vagina and saline wash given. Injection prochlorperazine and hydrocortisone was administered intravenously immediately. After half an hour patient developed fever $\left(100.4^{\circ} \mathrm{F}\right)$, however the rash disappeared in 6 to 8 hours. Patient developed slight bleeding per vaginum within 3 hours of instillation the drug. She had complete expulsion of the abortus within 6 hours of intravaginal administration of misoprostol. She was started on antihistamines and corticosteroids (prednisolone $40 \mathrm{mg}$ ) which were tapered off gradually in 20 days. Her biochemical and hematologic profile before and after this episode revealed no abnormality and she was discharged in satisfactory condition on second day of admission.

\section{Comment}

We have searched the pubmed/medline using the terms "misoprostol” AND "hypersensitivity reaction”/“allergic 
reaction”. Lichenoid eruptions caused by misoprostol has been reported by Cruz MJ et al. [3]. A case of anaphylactic shock and mycotic necrosis after treatment with Artotec, a combination of diclofenac sodium with misoprostol has been reported [4]. On the contrary various studies have reported protective effect of misoprostol in allergic diseases. Babakhin AA et al. has shown that misoprostol can inhibit basophil histamine release indicating a potentially beneficial role of PGE1 analogs as pharmacotherapy for allergic diseases [5]. Inoue $\mathrm{Y}$ et al. has shown that misoprostol may suppress the absorption of the allergen levels and outbreak the allergic symptom induced by aspirin in the patients with WDEIA (wheat dependent exercise induced anaphylaxis) [6]. In our case the patient had a severe hypersensitivity reaction with intravaginal administration of misoprostol hence possibility of hypersensitivity reaction must be kept in mind while using misoprostol.

\section{Disclosure Statement}

There is no conflict of interest among the authors.

\section{REFERENCES}

[1] C. Fiala and K. Gemzell-Danielsson, "Review of Medical
Abortion Using Mifepristone in Combination with Prostaglandin Analogue,” Contraception, Vol. 74, No. 1, 2006, pp. 66-86. doi:10.1016/j.contraception.2006.03.018

[2] New US Food and Drug Administration Labeling on Cytotec (Misoprostol) Use and Pregnancy, "ACOG Committee Opinion 283,” American College of Obstetricians and Gynecologists, Washington DC, 2003.

[3] M. J. Cruz, A. F. Duarte, T. Baudtier, A. P. Cunha, F. Barreto and F. Azevedo, "Lichenoid Drug Eruption Induced by Misoprostol,” Contact Dermatitis, Vol. 61, No. 4, 2009, pp. 240-242. doi:10.1111/j.1600-0536.2009.01616.x

[4] C. Meuleman, P. Jourdain, M. Bellorini, N. Guillard, J. Loiret, B. Thebault, et al., "Anaphylactic Shock and Myocytic Necrosis after Treatment with Artotec," Archives des Maladies du Coeur et des Vaisseaux, Vol. 95, No. 12, 2002, pp. 1230-1233.

[5] A. A. Babakhin, H. Nolte and L. M. DuBuske, "Effect of Misoprostol on the Secretion of Histamine from Basophils of Whole Blood," Annals of Allergy, Asthma \& Immunology. Vol. 84, No. 3, 2000, pp. 361-365. doi:10.1016/S1081-1206(10)62787-1

[6] Y. Inoue, A. Adachi, M. Ueno, et al., “The Inhibition Effect of a Synthetic Analogue of Prostaglandin E1 to the Provocation by Aspirin in the Patients of WDEIA," Arerugi, Vol. 58, No. 10, 2009, pp. 1418-1425. 